\begin{tabular}{|c|l|}
\hline Title & Picosecond rotation of a ring-shaped optical lattice by using a chirped vortex-pulse pair \\
\hline Author(s) & Y amane, Keisaku; Sakamoto, Moritsugu; Murakami, Naoshi; Morita, Ryuji; Oka, Kazuhiko \\
\hline Citation & $\begin{array}{l}\text { Optics Letters, 41(19), 4597-4600 } \\
\text { https://doi.org/40.1364/0L.41.004597 }\end{array}$ \\
\hline Issue Date & 2016-09-03 \\
\hline Doc URL & http://hdl.handle.net/2115/67098 \\
\hline Rights & $\begin{array}{l}\text { ○ 2013 Optical Society of A merica. One print or electronic copy may be made for personal use only. Systematic } \\
\text { reproduction and distribution, duplication of any material in this paper for a fee or for commercial purposes, or } \\
\text { modifications of the content of this paper are prohibited }\end{array}$ \\
\hline Type & article (author version) \\
\hline File Information & \begin{tabular}{l} 
OP_41_4597.pdf \\
\hline
\end{tabular} \\
\hline
\end{tabular}

Instructions for use 


\title{
Picosecond rotation of ring-shaped optical lattice by using chirped vortex-pulse pair
}

\author{
K. Yamane ${ }^{1}$, M. Sakamoto ${ }^{2,3}$, N. Murakami ${ }^{1}$, R. Morita ${ }^{1}$, and K. OKa ${ }^{1, *}$ \\ ${ }^{1}$ Division of Applied Physics, Faculty of Engineering, Hokkaido University, Sapporo, Hokkaido 060-8628, Japan \\ ${ }^{2}$ Division of Applied Physics, Graduate School of Engineering, Hokkaido University, Sapporo, Hokkaido 060-8628, Japan \\ ${ }^{3}$ Department of Electrical Engineering, Nagaoka University of Technology, 1603-1 Kamitomioka, Nagaoka, Niigata 940-2188, Japan \\ ${ }^{*}$ Corresponding author: oka@eng.hokudai.ac.jp
}

Compiled September 3, 2016

\begin{abstract}
A novel method of ultrafast rotation of ring-shaped optical lattice in the picosecond time region was proposed and demonstrated. Our ring-lattice generator was assembled by a pair of linearly chirped pulses with time delay, a high-order birefringent retarder, and an axially symmetric polarization element. Using a mode-locked Ti:sapphire laser oscillator as a light source, stable two-, four-, and six-petaled ring-lattice rotations were respectively demonstrated with the rotation periods of $1.6 \mathrm{ps}$, $3.2 \mathrm{ps}$, and $4.8 \mathrm{ps}$. Our method has a potential to open up a new technique to resonantly excite propagating quasi-particles together with their coherent enhancement. (-) 2016 Optical Society of America
\end{abstract}

OCIS codes: (050.4865) Optical vortices; (140.3300) Laser beam shaping; (260.6042) Singular optics; (320.1590) Chirping; (320.5390) Picosecond phenomena; (320.7100) Ultrafast measurements.

http://dx.doi.org/10.1364/OL.XX.XXXXXX

When two optical vortices (OVs) with different topological charges (TCs) $\ell_{1}$ and $\ell_{2}$ coaxially interfere with each other, a ring-shaped optical lattice is generated [1,2]. This type of ring lattice has several interesting features that its spatial structure has $\left|\ell_{1}-\ell_{2}\right|$-fold rotational symmetry and that both bright and dark lattices can be realized by choosing appropriate pairs of $\ell_{1}$ and $\ell_{2}$. Moreover, the ring lattice can be easily rotated by controlling the relative phase difference between two OVs. Because of these attractive features, much attention has been paid for the generation and application of the ring-shaped optical lattice. Although the ring lattice has been mainly used in optical trapping [3] so far, its application areas can be expanded if we can improve the basic performance of the ring-lattice generation.

One of the key features of the ring-lattice generation is its capability for the rapid rotation; since the ring lattice can be rotated simply by changing the phase difference between two OVs, namely without rewriting the holograms on the spatial light modulators (SLMs) generating the respective OVs, the rotation speed can be raised up to the speed of phase modulation.
In previous studies on the ring-lattice generation, faster rotation has been achieved by use of optoelectronic devices. For example, Franke-Arnold et al. used acousto-optic modulators (AOMs) to introduce a frequency difference between two OVs generated by a SLM [2]. They experimentally demonstrated the ring-lattice rotation at the frequency of 10's of MHz. Alternatively, the present authors previously developed a method using an electro-optic modulator (EOM) combined with a vortex converter comprising axially symmetric polarization elements (ASPEs) [4]. Although these methods allow us to rotate the ring lattice much faster than the speed of direct rewriting of the SLMs, their rotation speed is still limited up to the order of $\mathrm{GHz}$ on account of the bandwidth restriction of the optoelectronic phase modulators. Meanwhile, if we can rotate the ring lattice in the ultrafast regime, it leads to a novel technique for resonantly exciting quasi-particles that rotate or propagate, with controllable rotating-velocity to satisfy the phasematching condition, and thereby enabling coherently enhancement of their propagation [5]. Under these circumstances, we conceived a novel method for the ultrafast rotation of a ringshaped optical lattice. Instead of using optoelectronic devices, the new method incorporates a chirped-pulse generator and a delay line so that time-shifted two chirped pulses are respectively converted to OVs with different TCs. Since the frequency difference between two OVs can be raised up to the order of $\mathrm{THz}$, the present method allows us to rotate the ring lattice in the picosecond regime, which cannot be achieved by the conventional methods using optoelectronic phase modulators.

The conceptual scheme of the optical ring-lattice generator capable of ultrafast rotation is illustrated in Fig. 1. An ultrashort laser pulse first passes through a pulse stretcher in which pulse duration of the incident optical pulse is expanded in time by a dispersive element such as an optical fiber, a pair of gratings, a pair of prisms, and a thick crystal. Owing to the dispersion of the dispersive element, the light emerging from the pulse stretcher is converted to a chirped pulse whose instantaneous angular-frequency continuously varies with time. Provided that the nonlinearity, and third and higher order dispersions of the dispersive element are negligible, the instantaneous angular frequency of the chirped pulse can be approximated as

$$
\omega(t)=\omega_{0}+C t
$$




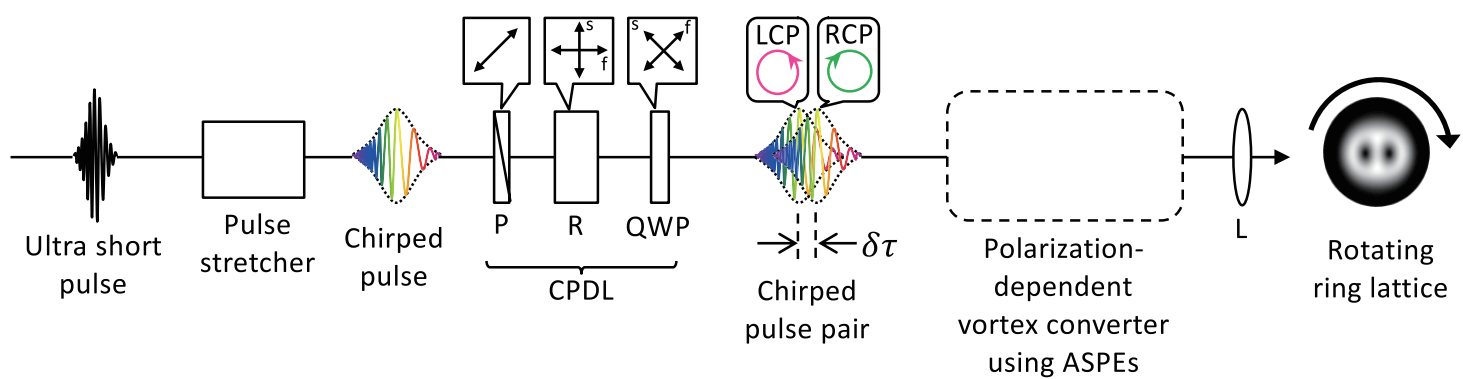

Fig. 1. Conceptual scheme of optical ring-lattice generator capable of ultrafast rotation.

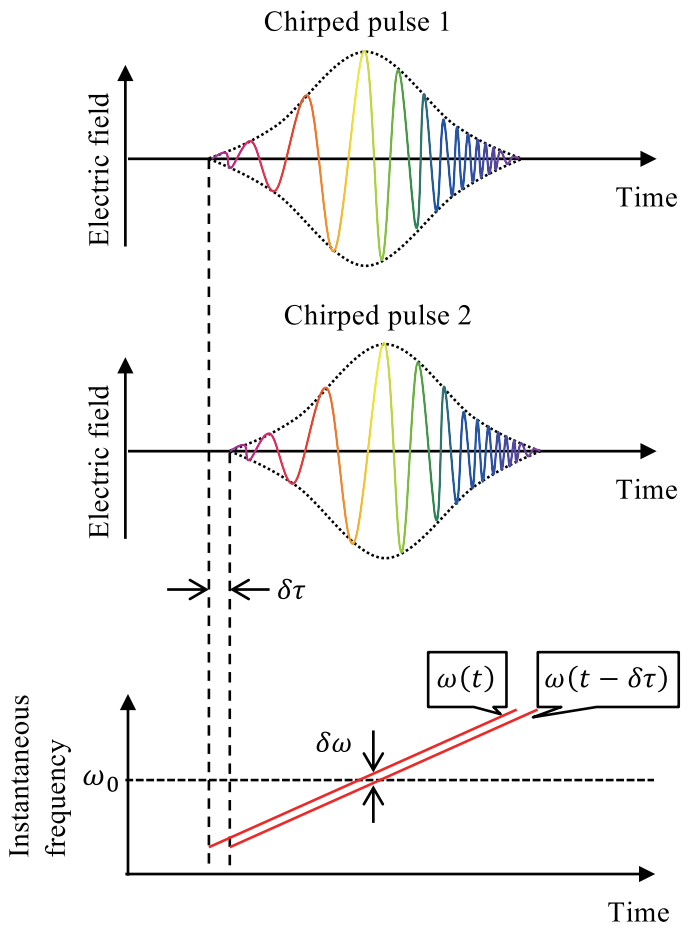

Fig. 2. Superposition of two chirped pulses and instantaneous frequency difference.

where $t$ is the time, $\omega_{0}$ is the central angular-frequency, and $C$ is the chirp coefficient. For a strongly chirped pulse, $C$ almost equals to the reciprocal of the group delay dispersion (GDD) of the dispersive element.

The chirped pulse from the pulse stretcher is then incident on a circular-polarization delay line (CPDL), consisting of a polarizer $\mathrm{P}$, a high-order birefringent retarder $\mathrm{R}$, and a quarterwave plate QWP. The fast axis of QWP is arranged at $45 \mathrm{deg}$. to that of $R$, whereas the transmission axis of $P$ is inclined to both principal axes of $R$. After passing through $P$, the chirped pulse is decomposed into orthogonal linearly polarized eigenmodes of $\mathrm{R}$, which propagate through $\mathrm{R}$ with different group velocities. The respective eigenmodes of $R$ are then converted to left- and right-circular polarizations, LCP and RCP, by QWP. As a result, the light emerging from the CPDL consists of time-shifted two chirped pulses that have orthogonal circular-polarization with each other. The time shift $\delta \tau$ between LCP and RCP components is given by

$$
\delta \tau=B_{g} D / c,
$$

where $B_{g}$ is the group birefringence of R, $D$ is its thickness, and $c$ is the speed of light in vacuum. As illustrated in Fig. 2, the (a)

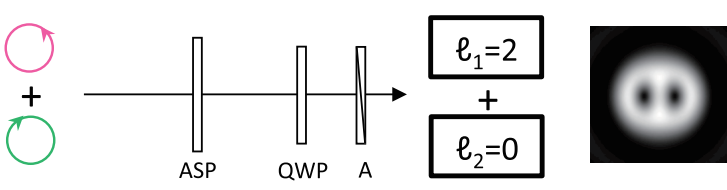

(b)

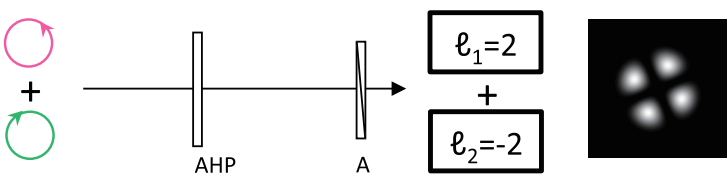

(c)

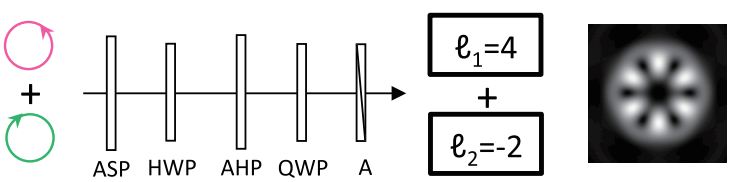

Fig. 3. Examples of vortex converter using ASPEs and the resultant ring lattices. (a), (b), and (c) are designed to generate TC pairs of $\left(\ell_{1}, \ell_{2}\right)=(2,0),(2,-2)$, and $(4,-2)$, respectively.

time shift $\delta \tau$ introduces constant angular-frequency difference $\delta \omega$ between paired chirped-pulses emerging from the CPDL, because the instantaneous angular frequencies of the chirped pulses are linearly related to time as Eq. (1). The angularfrequency difference $\delta \omega$ is related to the time shift $\delta \tau$ as

$$
\delta \omega=\omega(t)-\omega(t-\delta \tau)=C \delta \tau .
$$

The time-shifted chirped-pulse pair from CPDL is then launched into a polarization-dependent vortex converter comprising ASPEs [4]. ASPEs are space-variant polarization elements whose principal axes are axially distributed on the element, such as an axially symmetric half-wave plate (AHP) and an axially symmetric polarizer (ASP). Each ASPE has a function to shift the TC of an incident circularly polarized OV, and the sign of the TC-shift depends on the handedness of the incident circular polarization. By using the features of ASPEs, the vortex converter transforms the LCP and RCP components of the incident time-shifted chirped-pulse pair into two OVs with different TCs $\ell_{1}$ and $\ell_{2}$, respectively. Various pairs of even TCs can be realized by properly selecting the configuration of the vortex converter. Some typical examples are shown in Fig. 3; in this figure, (a), (b), and (c) are designed to generate TC pairs of $\left(\ell_{1}, \ell_{2}\right)=(2,0),(2,-2)$, and $(4,-2)$, respectively. Here HWP and $A$ respectively denote a half-wave plate and an analyzer.

The two OVs emerging from the vortex converter are finally interfere with each other at the back focal plane of a convex 
lens $\mathrm{L}$ in Fig. 1 to form a ring lattice. Since the interfering two OVs have angular-frequency difference of $\delta \omega$, the resultant ring lattice rotates at the period of

$$
T=\frac{2 \pi\left|\ell_{1}-\ell_{2}\right|}{\delta \omega}=\frac{2 \pi\left|\ell_{1}-\ell_{2}\right|}{C \delta \tau} .
$$

We here note that the angular-frequency difference $\delta \omega$ introduced by the present method can be increased up to the order of $\mathrm{THz}$, as will be demonstrated below. Such an ultrafast modulation cannot be realized by use of ordinal optoelectronic devices, such as AOMs and EOMs. Therefore, the present method allows us to rotate the optical ring lattice with the speed much faster than conventional methods.

The feasibility of present method was demonstrated by the experiment. As a light source emitting ultrashort pulses, we used a mode-locked Ti:sapphire laser oscillator (center wavelength: $\sim 800 \mathrm{~nm}$, repetition rate: $\sim 80 \mathrm{MHz}$, full width at half maximum (FWHM) of spectrum: $\sim 40 \mathrm{~nm}$, the average power: $\sim 200 \mathrm{~mW}$ ). A fused silica single-mode fiber of $1 \mathrm{~m}$ length was used as a pulse stretcher. The CPDL was assembled by use of a quartz high-order retarder R of length $D=13 \mathrm{~mm}$ and a FresnelRhomb type QWP. We here note that achromatic QWPs and HWPs, i.e. Fresnel Rhombs and composite waveplates, were used in the CPDL and the vortex converter to avoid the unwanted effect due to the retardation change within the wide spectral range of the ultrashort laser pulse. The chirp coefficient $C$ in Eq. (1) is evaluated to be $1.96 \times 10^{25} \mathrm{~s}^{-2}$ by considering GDDs of not only the fiber but all the other elements in the system. Meanwhile, the time delay $\delta \tau$ introduced by the CPDL is calculated to be 0.41 ps from Eq. (2), and thus the angularfrequency difference $\delta \omega$ in Eq. (3) is evaluated to be $8.0 \times 10^{12}$ $\operatorname{rad~s}^{-1}$. The duration of the chirped pulse was measured to be $\sim 6$ ps.

For the first experiment, the vortex converter of Fig. 3(a) was assembled by use of an ASP made of photonic crystal (Photonic Lattice Inc.) so that an OV with $\ell_{1}=2$ and a quasi-plane wave $\left(\ell_{2}=0\right)$ interfere with each other to generate a two-petaled ring lattice. The ASP has concentric, circular patterns that are continuous and not segmented [6]. The rotation period of the resultant lattice is evaluated from Eq. (4) to be 1.6 ps. We here note that the visibility of the generated ring lattice depends on the amplitude ratio between two OVs at the back focal plane of the convex-lens L, which can be controlled by the angle between the transmission axis of $\mathrm{P}$ and the fast axis of $\mathrm{R}$ in the CPDL. For the present experiment, the angle was set to be $21 \mathrm{deg}$. to have the maximum visibility for the case $\left(\ell_{1}, \ell_{2}\right)=(2,0)$. To observe the ultrafast rotation of the generated ring lattice, we conducted the time-resolved measurement using sum-frequency generation (SFG) between the chirped pulse pair and an ultrashort Gaussian probe pulse. As a nonlinear crystal for SFG, we use Type-I $10 \mu \mathrm{m}$-thick $\beta$ - $\mathrm{BaB}_{2} \mathrm{O}_{4}$ (BBO) crystal. The time-resolved SFG images are shown in Fig. 4 as a function of time delay in SFG with respect to an arbitrary origin. At each instant of time, a two-petaled SFG image that resembles the numerically simulated one is obtained. With the evolution of time, the twopetaled pattern rotates around optical axis without changing its pattern except rotation angles. The rotation period of the generated lattice is about $1.6 \mathrm{ps}$, which coincides with the value calculated from Eq. (4).

We next generated two other ring lattices by use of the vortex converters of Figs. 3(b) and 3(c). In respective cases, as shown in the right-hand sides of these figures, four- and six-petaled ring lattices should be generated from the OV pairs of $\left(\ell_{1}, \ell_{2}\right)=$

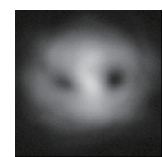

$0.0 \mathrm{ps}$

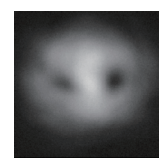

$0.8 \mathrm{ps}$

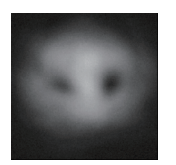

$1.6 \mathrm{ps}$

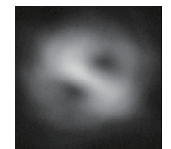

$0.2 \mathrm{ps}$

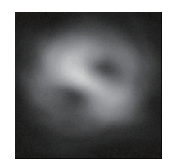

$1.0 \mathrm{ps}$

Numerically simulated lattice

pattern

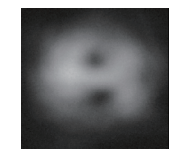

$1.2 \mathrm{ps}$

$1.4 \mathrm{ps}$
Fig. 4. Time-resolved SFG images as a function of time delay between the generated ring lattice and the probe pulse.

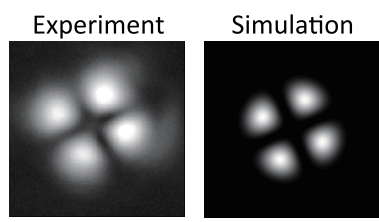

(a) $\ell_{1}=2$ and $\ell_{2}=-2$

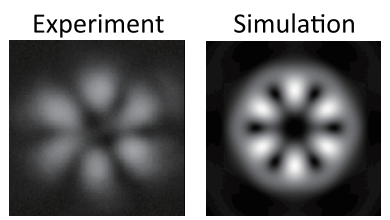

(b) $e_{1}=4$ and $e_{2}=-2$
Fig. 5. Examples of SFG images obtained with the TC combinations of (a) $\ell_{1}=2$ and $\ell_{2}=-2$, and (b) $\ell_{1}=4$ and $\ell_{2}=-2$. See Visualizations 1 and 2 for the image variations with respect to time delay for respective conditions.

$(2,-2)$ and $(4,-2)$. An AHP also made of photonic crystal that has concentric, circular patterns [6] was used in the assembly of these vortex converters. To obtain the maximum visibilities in the generated ring lattices, the angle between the transmission axis of $\mathrm{P}$ and the fast axis of $\mathrm{R}$ in the CPDL was set to be $45 \mathrm{deg}$. and $35 \mathrm{deg}$., respectively, for the cases with $\left(\ell_{1}, \ell_{2}\right)=$ $(2,-2)$ and $(4,-2)$. The time-resolved SFG images of four- and six-petaled ring lattices as functions of time delay in SFG are shown in the movies in Visualizations 1 and 2, respectively. In addition, examples of the SFG images for both ring lattices are shown in Figs. 5(a) and 5(b), together with the numerically simulated ones. Both SFG images are similar to the corresponding lattice patterns obtained from numerical simulation, except for slight distortions. The distortions can be attributed to the small imperfections of the experimental system, such as wavefront distortion of the reference wave and astigmatism due to the off-axis configuration of the SFG system. With the evolution of time, each SFG image rotates almost preserving its spatial pattern. The rotation periods of the four- and six-petaled patterns obtained from the movies in Visualizations 1 and 2 are 3.2 ps and $4.8 \mathrm{ps}$, respectively. They almost coincide with the periods evaluated from Eq. (4), i.e. 3.1 ps and 4.7 ps. Note that the timeresolved SFG images in Fig. 4, and Visualizations 1 and 2 are not normalized. In Visualization 2, the temporal variation in the intensity of the images is observed to be in accordance with the temporal envelope of the chirped pulses, while the spatial profile of the images almost keeps its shape except for the rotation of the lattice pattern. 
As demonstrated by the experiment, the present method allows us to rotate the ring lattice in the picosecond regime. Compared with the previous methods using AOMs and EOMs, the ring lattice is rotated at an extremely high speed because the angular-frequency difference $\delta \omega$ between interfering two OVs can be increased up to the order of $\mathrm{THz}$. By optimizing the parameters of the experimental system, faster rotation, namely the rotation with the sub-picosecond period, should also be realizable. We here note that the idea using the linear frequency sweep to introduce a frequency shift between two waves, described in Fig. 2, has a similarity with the technique of the frequency-modulated continuous wave (FMCW) for laser sensing [7]. The FMCW technique is usually assembled with a directly frequency-modulated laser, such as a continuous wave (CW) semiconductor laser, to generate a beat signal in $\mathrm{MHz}$ or $\mathrm{GHz}$ region. In contrast, the present method incorporates the chirped laser pulse instead of a CW laser, so that the frequency difference in $\mathrm{THz}$ region can be introduced. In other words, the present method is one of the realization of the FMCW technique within the ultrafast region.

To our knowledge, the present study is the first demonstration of the beam-profile rotation of an optical pulse in the ultrafast regime. Although the temporal modulation of the optical pulse has been extensively studied so far, almost no study has been made for the ultrafast spatial modulation of the beam profile because of the difficulty in the rapid direct-deformation of a beam pattern. The reason why the present method allows us to modulate the spatial beam profile in the picosecond regime is that the spatial modulation of the beam profile is realized by the interference between two temporally modulated OVs with different spatial phase distributions. Hence, the present method has a potential to open up new fields where the ultrafast spatial modulation of the laser beam is essential.

We here note that the present method requires an ultrafast laser pulse and thus the lattice can be rotated within the temporal width of the chirped pulse. Although the rotations within the pulse duration of our experimental demonstration were limited to one or a few cycles because of the restriction of our existing apparatus, this restriction can be relaxed if a pulse stretcher with a much stronger GDD is used; in that case, the chirped-pulse will be wide enough to contain much more rotations, such as several tens of rotations. In addition, even when lattice rotation is limited to only a few cycles, it is long enough to perform ultrafast measurements, for example, pump-probe measurements.

For the ultrafast rotation of the ring lattice by use of a chirped pulse, the vortex converter using ASPEs has several merits. The first merit is that the dispersion of the vortex generation using ASPEs is quite small $[6,8,9]$, because the wavefront conversion is made based on geometric phase. The ultrashort pulses, including chirped pulses, have considerably wide spectrum and thus the vortex converter should be achromatic within the spectral bandwidth of the chirped pulse. While other kinds of vortex generators using SLMs or spiral phase plates (SPPs) can also be used with a ultrashort pulse provided that their dispersions are well compensated [10], the vortex generation using ASPEs does not require dispersion compensation, particularly within the bandwidth of $\pm 20 \mathrm{~nm}$ of the present experiment. The second merit is that the vortex generation using ASPEs allows us to configure stable optical system. Since two OVs are coaxially converted from LCP and RCP components in the chirped-pulse pair, the system of Fig. 1 works as a polarization-separated common-path interferometer. It was confirmed by our experiments that the resultant ring lattice is stable and almost immune from external perturbations such as vibration. Therefore the vortex converter using ASPEs is suited for the ultrafast rotation of the ring lattices.

As an example for application, we here describe the coherent excitation enhancement of a quasi-particle by the ultrafast rotating ring-shaped lattices. When an exciton-polariton at 1.94 $\mathrm{eV}$ in a polydiacetylene crystal [11] is excited by a rotating ringshaped lattice with a rotating frequency of $\sim 1 \mathrm{THz}$ and a radius of $\sim 170 \mu \mathrm{m}$, which is composed of broadband chirped pulses with a central energy of $\sim 1.94 \mathrm{eV}(\sim 640 \mathrm{~nm})$ and a bandwidth of $\sim 120 \mathrm{meV}(\sim 40 \mathrm{~nm})$, the rotating velocity of the ring-shaped lattice is $\sim c / 1.5$ ( $c$ : velocity of light in vacuum). Since the rotating velocity of the ring-shaped lattice is greater than the phase velocity of the exciton-polariton $(\sim \mathcal{c} / 1.8)$ [11], the quasi-particle excitation can be coherently enhanced under the condition of Čerenkov radiation [12], resulting in coherent Archimedes spiral waves in the inner and outer directions in the cross section of the ring-shaped lattice. The wave front of such waves is expressed by $\rho-\rho_{0}= \pm a \phi$ in the cylindrical coordinates ( $a$, constant factor, $\rho_{0}$, constant radius).

In conclusion, we proposed and demonstrated a new method for the ultrafast rotation of an optical ring lattice. A chirped-pulse generator and a circular-polarization delay line are incorporated in the optical system, so that the angularfrequency difference $\delta \omega$ between interfering two OVs can be increased up to the order of THz. The polarization-dependent vortex converter using ASPEs is employed to avoid the dispersion effect as well as to enhance the stability against the external perturbations.

\section{FUNDING.}

JSPS KAKENHI Grant Numbers JP26286056, JP14J01485, JP15H04011, and JP16H06506; Commissioned Research of National Institute of Information and Communications Technology (NICT), JAPAN.

\section{REFERENCES}

1. L. Amico, A. Osterloh, and F. Cataliotti, Phys. Rev. Lett. 95, 063201 (2005).

2. S. Franke-Arnold, J. Leach, M. J. Padgett, V. E. Lembessis, D. Ellinas, A. J. Wright, J. M. Girkin, P. Öhberg, and A. S. Arnold, Opt. Express 15, 8619 (2007).

3. L. Paterson, M. P. MacDonald, J. Arlt, W. Sibbett, P. E. Bryant, and K. Dholakia, Science 292, 912 (2001).

4. M. Sakamoto, K. Oka, R. Morita, and N. Murakami, Opt. Lett. 38, 3661 (2013).

5. I. V. Dzedolik and V. Pereskokov, J. Opt. Soc. Am. A 33, 1004 (2016).

6. N. Murakami, S. Hamaguchi, M. Sakamoto, R. Fukumoto, A. Ise, K. Oka, N. Baba, and M. Tamura, Opt. Express 21, 7400 (2013).

7. D. Uttam and B. Culshaw, IEEE Journal of Lightwave Technology 3, 971 (1985).

8. M. Sakamoto, R. Fukumoto, N. Murakami, R. Morita, and K. Oka, Opt. Rev. 22, 174 (2015).

9. K. Yamane, Z. Yang, Y. Toda, and R. Morita, New Journal of Physics 16, 053020 (2014).

10. M. Bock, J. Jahns, and R. Grunwald, Opt. Lett. 37, 3804 (2012).

11. A. I. Gutman and A. V. Sel'kin, Phys. Lett. A 158, 158 (1991).

12. P. K. Tien, R. Ulrich, and R. J. Martin, Appl. Phys. Lett. 17, 447 (1970). 


\section{FULL REFERENCES}

1. L. Amico, A. Osterloh, and F. Cataliotti, "Quantum many particle systems in ring-shaped optical lattices," Phys. Rev. Lett. 95, 063201 (2005).

2. S. Franke-Arnold, J. Leach, M. J. Padgett, V. E. Lembessis, D. Ellinas, A. J. Wright, J. M. Girkin, P. Öhberg, and A. S. Arnold, "Optical ferris wheel for ultracold atoms," Opt. Express 15, 8619-8625 (2007).

3. L. Paterson, M. P. MacDonald, J. Arlt, W. Sibbett, P. E. Bryant, and K. Dholakia, "Controlled rotation of optically trapped microscopic particles," Science 292, 912-914 (2001).

4. M. Sakamoto, K. Oka, R. Morita, and N. Murakami, "Stable and flexible ring-shaped optical-lattice generation by use of axially symmetric polarization elements," Opt. Lett. 38, 3661-3664 (2013).

5. I. V. Dzedolik and V. Pereskokov, "Formation of vortices by interference of surface plasmon polaritons," J. Opt. Soc. Am. A 33, 1004-1009 (2016).

6. N. Murakami, S. Hamaguchi, M. Sakamoto, R. Fukumoto, A. Ise, K. Oka, N. Baba, and M. Tamura, "Design and laboratory demonstration of an achromatic vector vortex coronagraph," Opt. Express 21, 7400-7410 (2013).

7. D. Uttam and B. Culshaw, "Precision time domain reflectometry in optical fiber systems using a frequency modulated continuous wave ranging technique," IEEE Journal of Lightwave Technology 3, 971-977 (1985).

8. M. Sakamoto, R. Fukumoto, N. Murakami, R. Morita, and K. Oka, "Dispersion reduction in generation of high-order optical vortex using axially symmetric half-wave plates," Opt. Rev. 22, 174-178 (2015).

9. K. Yamane, Z. Yang, Y. Toda, and R. Morita, "Frequency-resolved measurement of the orbital angular momentum spectrum of femtosecond ultra-broadband optical-vortex pulses based on field reconstruction," New Journal of Physics 16, 053020 (2014).

10. M. Bock, J. Jahns, and R. Grunwald, "Few-cycle high-contrast vortex pulses," Opt. Lett. 37, 3804-3806 (2012).

11. A. I. Gutman and A. V. Sel'kin, "Exciton polaritons in polydiacetylenets crystals," Phys. Lett. A 158, 158-160 (1991).

12. P. K. Tien, R. Ulrich, and R. J. Martin, "Optical second harmonic generation in form of coherent cerenkov radiation from a thin - film waveguide," Appl. Phys. Lett. 17, 447-449 (1970). 